\title{
Numerical dynamics of a nonstandard finite-difference- $\theta$ method for a red blood cell survival model
}

Yuanyuan Wang*

\begin{tabular}{l}
\hline${ }^{*}$ Correspondence: \\
y-y-wang@163.com; \\
wangyy@upc.edu.cn \\
College of Science, China University \\
of Petroleum (East China), Qingdao, \\
266580, P.R. China
\end{tabular}

266580, P.R. China

\begin{abstract}
In this article, by a nonstandard finite-difference- $\theta$ (NSFD- $\theta$ ) method we study the dynamics of a discrete red blood cell survival model. Firstly, the linear stability of the model is discussed. It is found that the Neimark-Sacker bifurcation exists when the delay passes a sequence of critical values. Then the explicit algorithm for determining the direction of the Neimark-Sacker bifurcation and the stability of the bifurcating periodic solutions are derived by using the normal form method and center manifold theorem. Our results show the NSFD- $\theta$ method could inherit the Hopf bifurcation and the asymptotically stability for sufficiently small step-size $h=1 / m$, where $m$ is a positive integer. In particular, for $\theta=0,1 / 2,1$ the results hold for any step-size $h=1 / \mathrm{m}$. Finally, numerical examples are provided to illustrate the theoretical results.
\end{abstract}

Keywords: nonstandard finite-difference- $\theta$ (NSFD- $\theta$ ) method; delay; Neimark-Sacker bifurcation; Hopf bifurcation

\section{Introduction}

In order to describe the survival of red blood cells in animal, Wazewska-Czyzewska and Lasota [1] proposed the following autonomous functional differential equation:

$$
\frac{d x}{d t}=-a x(t)+b e^{-c x(t-\tau)}
$$

where $a \in(0,1), b, c, \tau \in(0, \infty)$. Here $x(t)$ denotes the number of red blood cells at time $t$, $a$ is the probability of death of a red blood cell, $b$ and $c$ are positive constants related to the production of red blood cells per unit time, and $\tau$ is the time required to produce a red blood cell. A great deal of research has been devoted to the asymptotic behavior, periodic solutions, global attractivity, and other properties of this model.

The bifurcations of continuous-time model have been discussed in [2-5]. But due to scientific computation and simulation, our interest is focused on the behavior of discrete dynamical system corresponding to (1.1). It is desired that the discrete-time model is 'dynamically consistent' with the continuous-time model. In [6-12], for many numerical methods, e.g. Runge-Kutta methods and linear multistep methods, it has been proved that they could retain the local stability and the Hopf bifurcation for some delay differential equations and integro-differential equations.

(c) 2015 Wang; licensee Springer. This is an Open Access article distributed under the terms of the Creative Commons Attribution License (http://creativecommons.org/licenses/by/4.0), which permits unrestricted use, distribution, and reproduction in any medium, provided the original work is properly credited. 
In [13, 14], Wulf and Ford showed that, if applying the Euler forward method to solve the delay differential equation, then the discrete scheme is 'dynamically consistent.' It means that for all sufficiently small step-sizes the discrete model undergoes a Hopf bifurcation of the same type as the original model. The NSFD scheme [15-18] tries to preserve the significant properties of their continuous analogs and consequently gives reliable numerical results. In [19], by the $\theta$-method Tian investigated the stability properties for the solution of delay differential equations. In [6] by using the Euler method a discrete red blood cell survival model has been discussed. In this paper, we apply a nonstandard finite-difference- $\theta$ (NSFD- $\theta$ ) method to discretize the red blood cell survival model (1.1) with delay. The NSFD- $\theta$ scheme is a combination of the NSFD scheme and the $\theta$ method. So we construct a more general NSFD scheme for model (1.1). We obtain the consistent dynamical results of the corresponding continuous-time model by the NSFD- $\theta$ method for sufficiently small step-size. In particular, for $\theta=0,1 / 2,1$ the results hold for any step-size.

The paper is organized as follows. In Section 2, we analyze the distribution of the characteristic equation associated with the discrete red blood cell survival model, and we obtain the existence of the Neimark-Sacker bifurcation. In Section 3, the direction and stability of periodic solutions bifurcating from the Neimark-Sacker bifurcation of the discrete red blood cell model are determined by using the theories of discrete systems. In the final section, some computer simulations are performed to illustrate the analytical results.

\section{Stability analysis}

Let $u(t)=x(\tau t)$. Then (1.1) can be rewritten as

$$
\frac{d u}{d t}=-a \tau u(t)+b \tau e^{-c u(t-1)} .
$$

The differential equation

$$
\frac{d u}{d t}=-a \tau u(t)
$$

has the general solution $u(t)=\bar{C} e^{-a \tau t}$. We consider a step-size of the form $h=1 / m$, where $m \in Z_{+}$. The solution can be written as

$$
\frac{u(t+h)-u(t)}{\frac{1-e^{-a \tau h}}{a \tau}}=-a \tau u(t) .
$$

This is an exact finite-difference numerical method,

$$
\begin{aligned}
u(t+h)-u(t) & =\bar{C} e^{-a \tau(t+h)}-\bar{C} e^{-a \tau t} \\
& =\bar{C} e^{-a \tau t}\left(e^{-a \tau h}-1\right) \\
& =-a \tau u(t) \frac{1-e^{-a \tau h}}{a \tau} .
\end{aligned}
$$

Employ the NSFD- $\theta$ method to (2.1) and choose the 'denominator function' $\phi$ as

$$
\phi(h)=\frac{1-e^{-a \tau h}}{a \tau} .
$$


It yields the difference equation

$$
u_{n+1}=e^{-a \tau h} u_{n}+\frac{b}{a}\left(1-e^{-a \tau h}\right)\left[(1-\theta) e^{-c u_{n-m}}+\theta e^{-c u_{n+1-m}}\right]
$$

One can see that if $u_{*}$ is a fixed point to (2.3), then $u_{*}$ satisfies

$$
a u_{*}=b e^{-c u_{*}} .
$$

Set $y_{n}=u_{n}-u_{*}$. Then $y_{n}$ satisfies

$$
y_{n+1}=e^{-a \tau h} y_{n}+\left(e^{-a \tau h}-1\right) u_{*}+\frac{b\left(1-e^{-a \tau h}\right)}{a}\left[(1-\theta) e^{-c\left(y_{n-m}+u_{*}\right)}+\theta e^{-c\left(y_{n-m+1}+u_{*}\right)}\right] \text {. }
$$

Introducing a new variable $Y_{n}=\left(y_{n}, y_{n-1}, \ldots, y_{n-m}\right)^{T}$, we can rewrite (2.5) as

$$
Y_{n+1}=F\left(Y_{n}, \tau\right)
$$

where $F=\left(F_{0}, F_{1}, \ldots, F_{m}\right)^{T}$ and

$$
F_{k}=\left\{\begin{aligned}
e^{-a \tau h} y_{n-k}+\left(e^{-a \tau h}-1\right) u_{*} & \\
+\frac{b\left(1-e^{-a \tau h}\right)}{a}\left[(1-\theta) e^{-c\left(y_{n-m-k}+u_{*}\right)}+\theta e^{-c\left(y_{n-m+1-k}+u_{*}\right)}\right], & k=0, \\
y_{n-k+1}, & k=1,2, \ldots, m .
\end{aligned}\right.
$$

Clearly the origin is a fixed point of map (2.6), and the linear part of map (2.6) is

$$
Y_{n+1}=A Y_{n}
$$

where

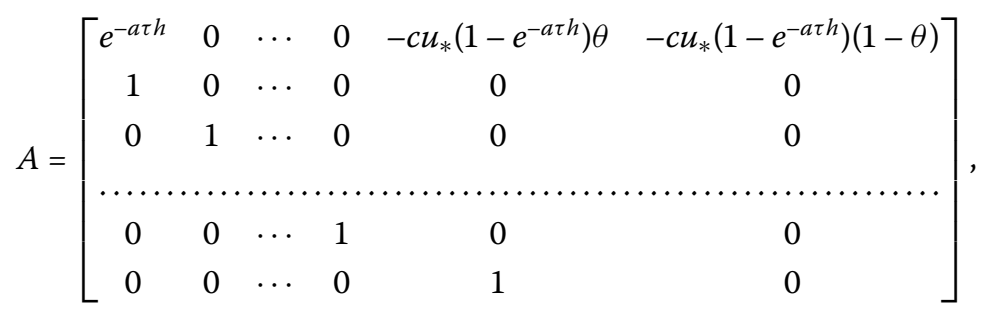

whose characteristic equation is

$$
\lambda^{m+1}-e^{-a \tau h} \lambda^{m}+c u_{*}\left(1-e^{-a \tau h}\right) \theta \lambda+c u_{*}\left(1-e^{-a \tau h}\right)(1-\theta)=0 .
$$

Lemma 1 All roots of (2.10) have modulus less than one for sufficiently small $\tau>0$.

Proof For $\tau=0,(2.10)$ becomes

$$
\lambda^{m+1}-\lambda^{m}=0 \text {. }
$$

The equation has an $m$-fold $\operatorname{root} \lambda=0$ and a simple root $\lambda=1$. 
Consider the root $\lambda(\tau)$ such that $\lambda(0)=1$. This root is a $C^{1}$ function of $\tau$. For (2.10), we have

$$
\frac{d|\lambda|^{2}}{d \tau}=\lambda \frac{d \bar{\lambda}}{d \tau}+\bar{\lambda} \frac{d \lambda}{d \tau},\left.\quad \frac{d|\lambda|^{2}}{d \tau}\right|_{\lambda=1, \tau=0}=-2 h a\left(1+c u_{*}\right)<0 .
$$

Consequently, all roots of (2.10) lie in $|\lambda|<1$ for sufficiently small $\tau>0$.

Lemma 2 For any step-size $h$, if $c u_{*}<1$, then (2.10) has no root with modulus one for all $\tau>0$.

Proof A Neimark-Sacker bifurcation occurs when two roots of the characteristic equation (2.10) cross the unit circle. We have to find values of $\tau$ such that there exist roots on the unit circle. The roots on the unit circle are given by $e^{\mathrm{i} w}, w \in(-\pi, \pi]$. Since we are dealing with a real polynomial, complex roots occur in complex conjugate pairs and we have only to look for $w \in(0, \pi]$. For $w \in(0, \pi], e^{\mathrm{i} w}$ is a root of $(2.10)$ if and only if

$$
\begin{aligned}
& e^{\mathrm{i}(m+1) w}-e^{-a \tau h} e^{\mathrm{i} m w}+c u_{*}\left(1-e^{-a \tau h}\right) \theta e^{\mathrm{i} w}+c u_{*}\left(1-e^{-a \tau h}\right)(1-\theta)=0 \\
& \left\{\begin{array}{c}
\cos (m+1) \omega-e^{-a \tau h} \cos m \omega+c u_{*}\left(1-e^{-a \tau h}\right) \theta \cos \omega \\
\quad+c u_{*}\left(1-e^{-a \tau h}\right)(1-\theta)=0 \\
\sin (m+1) \omega-e^{-a \tau h} \sin m \omega+c u_{*}\left(1-e^{-a \tau h}\right) \theta \sin \omega=0 .
\end{array}\right.
\end{aligned}
$$

Therefore

$$
\cos \omega=1+\frac{\left(1-e^{-a \tau h}\right)^{2}\left(1-\left(c u_{*}\right)^{2}\right)}{2\left[e^{-a \tau h}+\theta(1-\theta)\left(c u_{*}\left(1-e^{-a \tau h}\right)\right)^{2}\right]} .
$$

If $c u_{*}<1$, then $\cos w>1$, which yields a contradiction. So (2.10) has no root with modulus one for all $\tau>0$.

If $c u_{*}>1$, for any step-size $h,|\cos \omega|<1$ and $\tau>0$ is positive real, from (2.13) we know that

$$
\begin{aligned}
\omega_{k} & =\arccos \left(1+\frac{\left(1-e^{-a \tau h}\right)^{2}\left(1-\left(c u_{*}\right)^{2}\right)}{2\left[e^{-a \tau h}+\theta(1-\theta)\left(c u_{*}\left(1-e^{-a \tau h}\right)\right)^{2}\right]}\right)+2 k \pi, \\
k & =0,1,2, \ldots,\left[\frac{m-1}{2}\right]
\end{aligned}
$$

where [.] denotes the greatest integer function. It is clear that there exists a sequence of the time delay parameters $\tau_{k}$ satisfying (2.12) according to $\omega=\omega_{k}$.

Let $\lambda_{i}(\tau)=r_{i}(\tau) e^{\mathrm{i} w_{i}(\tau)}$ be a root of $(2.10)$ near $\tau=\tau_{i}$ satisfying $r_{i}\left(\tau_{i}\right)=1$ and $w_{i}\left(\tau_{i}\right)=w_{i}$. We have the following result.

Lemma 3 For sufficiently small step-size $h$, if $c u_{*}>1$, then

$$
\left.\frac{d r_{i}^{2}(\tau)}{d \tau}\right|_{\tau=\tau_{i}, w=w_{i}}>0
$$

Particularly, if $\theta=0,1 / 2,1$, the above results hold for any step-size $h$. 
Proof From (2.10), we obtain

$$
\begin{aligned}
& \lambda^{m}=\frac{\left(1-e^{-a \tau h}\right)\left(-c u_{*}\right)(\theta \lambda+1-\theta)}{\lambda-e^{-a \tau h}}, \\
& \left.\frac{d r_{i}^{2}(\tau)}{d \tau}\right|_{\tau=\tau_{i}, w=w_{i}}=\lambda \frac{d \bar{\lambda}}{d \tau}+\bar{\lambda} \frac{d \lambda}{d \tau}=\frac{2 a h e^{-a \tau h}}{1-e^{-a \tau h}} \frac{A C+B D}{C^{2}+D^{2}},
\end{aligned}
$$

where

$$
\begin{aligned}
& A=\theta \cos (2 w)+(1-2 \theta) \cos w-(1-\theta), \\
& B=\theta \sin (2 w)+(1-2 \theta) \sin w, \\
& C=\alpha \cos (2 w)-\beta \cos w+\gamma, \\
& D=\alpha \sin (2 w)-\beta \sin w \\
& \alpha=m \theta \\
& \beta=-[(m+1)(1-\theta)+\theta \exp (-a \tau h)(1-m)], \\
& \gamma=-m(1-\theta) \exp (-a \tau h), \\
& A C+B D=2(\theta \alpha+\theta \gamma-\alpha) \cos ^{2} w-(2 \theta-1)(\alpha+\beta+\gamma) \cos w+(2 \theta-1) \beta+\alpha-\gamma .
\end{aligned}
$$

Substituting (2.13) into (2.15), we obtain for sufficiently small step-size $h$

$$
\left.\frac{d r_{i}^{2}(\tau)}{d \tau}\right|_{\tau=\tau_{i}, w=w_{i}}=\lambda \frac{d \bar{\lambda}}{d \tau}+\bar{\lambda} \frac{d \lambda}{d \tau}>0
$$

Especially, when $\theta=0$

$$
\left.\frac{d r_{i}^{2}(\tau)}{d \tau}\right|_{\tau=\tau_{i}, w=w_{i}}=\frac{2 a h e^{-a \tau h}}{1-e^{-a \tau h}} \frac{\left(m+1+m e^{-a \tau h}\right)(1-\cos w)}{C^{2}+D^{2}}>0,
$$

when $\theta=1 / 2$

$$
\left.\frac{d r_{i}^{2}(\tau)}{d \tau}\right|_{\tau=\tau_{i}, w=w_{i}}=\frac{2 a h e^{-a \tau h}}{1-e^{-a \tau h}} \frac{0.5 m \sin ^{2} w\left(1+e^{-a \tau h}\right)}{C^{2}+D^{2}}>0,
$$

and when $\theta=1$

$$
\left.\frac{d r_{i}^{2}(\tau)}{d \tau}\right|_{\tau=\tau_{i}, w=w_{i}}=\frac{2 a h e^{-a \tau h}}{1-e^{-a \tau h}} \frac{(1-\cos w)\left(m+m e^{-a \tau h}\right)}{C^{2}+D^{2}}>0 .
$$

Therefore, if $\theta=0,1 / 2,1$, the results hold for any step-size $h$. This completes the proof.

Theorem 1 For system (2.6), the following statements are true:

(i) If $c u_{*}<1$, then $u=u_{*}$ is asymptotically stable for any $\tau>0$.

(ii) If $c u_{*}>1$, then $u=u_{*}$ is asymptotically stable for $\tau \in\left(0, \tau_{0}\right)$, and unstable for $\tau>\tau_{0}$. Equation (2.6) undergoes a Neimark-Sacker bifurcation at $u_{*}$ when $\tau=\tau_{i}$, for $i=0,1,2, \ldots,\left[\frac{m-1}{2}\right]$. 
Proof (i) If $c u_{*}<1$, from Lemmas 1 and 2, we know that (2.10) has no root with modulus one for all $\tau>0$. Applying Corollary 2.4 in [20], all roots of (2.10) have modulus less than one for all $\tau>0$. The conclusion follows.

(ii) If $c u_{*}>1$, applying Lemma 3, we know that all roots of (2.10) have modulus less than one when $\tau \in\left(0, \tau_{0}\right)$, and (2.10) has at least a couple of roots with modulus greater than one when $\tau>\tau_{0}$. The conclusion follows.

Remark 1 According to the conclusions of Theorem 1, we have results that are consistent with those for the corresponding continuous-time model (see [5]).

\section{Direction and stability of the Neimark-Sacker bifurcation in discrete model}

In the previous section, we obtained conditions for Neimark-Sacker bifurcation to occur when $\tau=\tau_{i}$, for $i=0,1,2, \ldots,\left[\frac{m-1}{2}\right]$. In this section we study the direction of the NeimarkSacker bifurcation and the stability of the bifurcating periodic solutions when $\tau=\tau_{0}$, using techniques from normal form and center manifold theory [21-23].

Denote

$$
\begin{aligned}
G\left(y_{n+1}, y_{n}, y_{n-m+1}, y_{n-m}\right)= & y_{n+1}-e^{-a \tau h} y_{n}-\left(e^{-a \tau h}-1\right) u_{*} \\
& -\frac{b\left(1-e^{-a \tau h}\right)}{a}\left[(1-\theta) e^{-c\left(y_{n-m}+u_{*}\right)}+\theta e^{-c\left(y_{n-m+1}+u_{*}\right)}\right] .
\end{aligned}
$$

Since $G(0,0,0,0)=0$ and $G_{y_{n+1}}(0,0,0,0)=1>0$, by the implicit function theory, there exists a function $y_{n+1}=\tilde{y}\left(y_{n}, y_{n-m+1}, y_{n-m}\right)$ satisfying $G\left(\tilde{y}\left(y_{n}, y_{n-m+1}, y_{n-m}\right), y_{n}, y_{n-m+1}, y_{n-m}\right)=$ 0 and

$$
\begin{aligned}
& \frac{\partial \tilde{y}\left(y_{n}, y_{n-m+1}, y_{n-m}\right)}{\partial y_{n}}=-\frac{G_{y_{n}}}{G_{y_{n+1}}}=e^{-a \tau h}, \\
& \frac{\partial \tilde{y}\left(y_{n}, y_{n-m+1}, y_{n-m}\right)}{\partial y_{n+1-m}}=-\frac{G_{y_{n+1-m}}}{G_{y_{n+1}}}=c u_{*}\left(1-e^{-a \tau h}\right) \theta e^{-c y_{n-m+1},} \\
& \frac{\partial \tilde{y}\left(y_{n}, y_{n-m+1}, y_{n-m}\right)}{\partial y_{n-m}}=-\frac{G_{y_{n-m}}}{G_{y_{n+1}}}=c u_{*}\left(1-e^{-a \tau h}\right)(1-\theta) e^{-c y_{n-m}} .
\end{aligned}
$$

Therefore

$$
\begin{aligned}
& \frac{\partial \tilde{y}(0,0,0)}{\partial y_{n}}=e^{-a \tau h}, \\
& \frac{\partial \tilde{y}(0,0,0)}{\partial y_{n+1-m}}=c u_{*}\left(1-e^{-a \tau h}\right) \theta, \\
& \frac{\partial \tilde{y}(0,0,0)}{\partial y_{n-m}}=c u_{*}\left(1-e^{-a \tau h}\right)(1-\theta) .
\end{aligned}
$$

In the sequence, we can calculate all the second-order and third-order partial derivatives of $\tilde{y}$ with respect to $y_{n}, y_{n-m+1}$ and $y_{n-m}$ at $(0,0,0)$, respectively. So, we can rewrite map (2.6) as

$$
Y_{n+1}=A Y_{n}+\frac{1}{2} B\left(Y_{n}, Y_{n}\right)+\frac{1}{6} C\left(Y_{n}, Y_{n}, Y_{n}\right)+O\left(\left\|Y_{n}^{4}\right\|\right)
$$


where

$$
\begin{aligned}
& B\left(Y_{n}, Y_{n}\right)=\left(b_{0}\left(Y_{n}, Y_{n}\right), 0, \ldots, 0\right)^{T}, \\
& C\left(Y_{n}, Y_{n}, Y_{n}\right)=\left(c_{0}\left(Y_{n}, Y_{n}, Y_{n}\right), 0, \ldots, 0\right)^{T} .
\end{aligned}
$$

Here $B(\phi, \psi)$ and $C(\phi, \psi, \eta)$ are symmetric multilinear vector functions of $\phi, \psi, \eta \in \mathbb{C}^{m+1}$. In coordinates we have

$$
\begin{aligned}
& b_{0}(\phi, \psi)=\left.\sum_{j, k=1}^{m+1} \frac{\partial^{2} g(\xi)}{\partial \xi_{j} \partial \xi_{k}}\right|_{\xi=0} \phi_{j} \psi_{k}, \\
& c_{0}(\phi, \psi, \eta)=\left.\sum_{j, k, l=1}^{m+1} \frac{\partial^{3} g(\xi)}{\partial \xi_{j} \partial \xi_{k} \partial \xi_{l}}\right|_{\xi=0} \phi_{j} \psi_{k} \eta_{l},
\end{aligned}
$$

in which $g(\xi)=\tilde{y}\left(\xi_{l}, \xi_{m}, \xi_{m+1}\right)$ for $\xi \in \mathbb{C}^{m+1}$.

Let $q=q\left(\tau_{0}\right) \in \mathbb{C}^{m+1}$ be an eigenvector of $A$ corresponding to $e^{\mathrm{i} w_{0}}$, then

$$
A q=e^{\mathrm{i} w_{0}} q, \quad A \bar{q}=e^{-\mathrm{i} w_{0}} \bar{q} .
$$

We also introduce an adjoint eigenvector $q^{*}=q^{*}(\tau) \in \mathbb{C}^{m+1}$ having the properties

$$
A^{T} q^{*}=e^{-\mathrm{i} w_{0}} q^{*}, \quad A^{T} \bar{q}^{*}=e^{\mathrm{i} w_{0}} \bar{q}^{*},
$$

and satisfying the normalization $\left\langle q^{*}, q\right\rangle=1$, where $\left\langle q^{*}, q\right\rangle=\sum_{i=0}^{m} \bar{q}_{i}^{*} q_{i}$.

Lemma 4 Define a vector valued function $q: \mathbb{C} \longrightarrow \mathbb{C}^{m+1}$ by

$$
p(\xi)=\left(\xi^{m}, \xi^{m-1}, \ldots, 1\right)^{T}
$$

If $\xi$ is an eigenvalue of $A$, then $A p(\xi)=\xi p(\xi)$.

In view of Lemma 4, we have

$$
q=p\left(e^{\mathrm{i} w_{0}}\right)=\left(e^{\mathrm{i} m w_{0}}, e^{\mathrm{i}(m-1) w_{0}}, \ldots, e^{\mathrm{i} w_{0}}, 1\right)^{T} .
$$

Lemma 5 Suppose $q^{*}=\left(q_{0}^{*}, q_{1}^{*}, \ldots, q_{m}^{*}\right)^{T}$ is the eigenvector of $A^{T}$ corresponding to the eigenvalue $e^{-\mathrm{i} w_{0}}$, and $\left\langle q^{*}, q\right\rangle=1$. Then

$$
q^{*}=\bar{K}\left(\frac{e^{\mathrm{i}(m-1) w_{0}}}{e^{-\mathrm{i} w_{0}}-e^{-a \tau h}}, e^{\mathrm{i}(m-1) w_{0}}, e^{\mathrm{i}(m-2) w_{0}}, \ldots, e^{2 \mathrm{i} w_{0}}, e^{\mathrm{i} w_{0}}, \frac{-c \mathcal{u}_{*}\left(1-e^{-a \tau h}\right)(1-\theta)}{e^{-\mathrm{i} w_{0}}-e^{-a \tau h}}\right)^{T},
$$

where $a_{m}=e^{-a \tau h}, a_{0}=-c u_{*}\left(1-e^{-a \tau h}\right)(1-\theta)$, and $a_{1}=-c u_{*}\left(1-e^{-a \tau h}\right) \theta$ are the coefficients of $\lambda$ in the characteristic equation (2.10), and

$$
K=\left[m-1+\frac{-c u_{*}\left(1-e^{-a \tau h}\right)(1-\theta) e^{-\mathrm{i}(m-1) w_{0}}}{1-e^{-\mathrm{i} w_{0}} e^{-a \tau h}}\right]^{-1} .
$$


Proof Assume $q^{*}$ satisfies $A^{T} q^{*}=\bar{z} q^{*}$ with $\bar{z}=e^{-\mathrm{i} w_{0}}$, then we have

$$
\left\{\begin{array}{l}
a_{m} q_{0}^{*}+q_{1}^{*}=\bar{z} q_{0}^{*}, \\
q_{k}^{*}=\bar{z} q_{k-1}^{*}, \quad k=2,3, \ldots, m-1, \\
a_{1} q_{0}^{*}+q_{m}^{*}=\bar{z} q_{m-1}^{*}, \\
a_{0} q_{0}^{*}=\bar{z} q_{m}^{*} .
\end{array}\right.
$$

Let $q_{m-1}^{*}=e^{\mathrm{i} w_{0}}$, by the normalization $\left\langle q^{*}, q\right\rangle=1$ and direct computation, the lemma follows.

Let $T_{\text {center }}$ denote a real eigenspace corresponding to $e^{ \pm i w_{0}}$, which is two-dimensional and is spanned by $\{\operatorname{Re}(q), \operatorname{Im}(\bar{q})\}$, and $T_{\text {stable }}$ be a real eigenspace corresponding to all eigenvalues of $A^{T}$, other than $e^{ \pm \mathrm{i} w_{0}}$, which is $(m-1)$-dimensional [23].

All vectors $x \in \mathbb{R}^{m+1}$ can be decomposed as

$$
x=v q+\bar{v} \bar{q}+y
$$

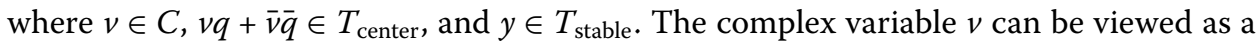
new coordinate on $T_{\text {center }}$, so we have

$$
v=\left\langle q^{*}, x\right\rangle, \quad y=x-\left\langle q^{*}, x\right\rangle q-\left\langle\bar{q}^{*}, x\right\rangle \bar{q}
$$

Let $a(\lambda)$ be characteristic polynomial of $A$ and $\lambda_{0}=e^{\mathrm{i} \omega_{0}}$, following the algorithms in [23] and using a computation process similar to $[13,14]$, we have

$$
\begin{aligned}
& g_{20}=\left\langle q^{*}, B(q, q)\right\rangle, \\
& g_{11}=\left\langle q^{*}, B(q, \bar{q})\right\rangle, \\
& g_{02}=\left\langle q^{*}, B(\bar{q}, \bar{q})\right\rangle, \\
& g_{21}=\left\langle q^{*}, B\left(\bar{q}, w_{20}\right)\right\rangle+2\left\langle q^{*}, B\left(q, w_{11}\right)\right\rangle+\left\langle q^{*}, C(q, q, \bar{q})\right\rangle,
\end{aligned}
$$

where

$$
\begin{aligned}
& w_{20}=\frac{b_{0}(q, q)}{a\left(\lambda_{0}^{2}\right)} p\left(\lambda_{0}^{2}\right)-\frac{\left\langle q^{*}, B(q, q)\right\rangle}{\lambda_{0}^{2}-\lambda_{0}} q-\frac{\left\langle\bar{q}^{*}, B(q, q)\right\rangle}{\lambda_{0}^{2}-\bar{\lambda}_{0}} \bar{q} \\
& w_{11}=\frac{b_{0}(q, \bar{q})}{a(1)} p(1)-\frac{\left\langle q^{*}, B(q, \bar{q})\right\rangle}{1-\lambda_{0}} q-\frac{\left\langle\bar{q}^{*}, B(q, \bar{q})\right\rangle}{1-\bar{\lambda}_{0}} \bar{q} .
\end{aligned}
$$

So, we can compute an expression for the critical coefficient $C_{1}\left(\tau_{0}\right)$ :

$$
C_{1}\left(\tau_{0}\right)=\frac{g_{20} g_{11}\left(1-2 \lambda_{0}\right)}{2\left(\lambda_{0}^{2}-\lambda_{0}\right)}+\frac{\left|g_{11}\right|^{2}}{1-\bar{\lambda}_{0}}+\frac{\left|g_{02}\right|^{2}}{2\left(\lambda_{0}^{2}-\bar{\lambda}_{0}\right)}+\frac{g_{21}}{2} \text {. }
$$

By (3.1), (3.2), and Lemma 5, we get

$$
\begin{aligned}
C_{1}\left(\tau_{0}\right)= & \frac{K}{2} \widetilde{c} \frac{e^{-\mathrm{i}(m-1) w_{0}}}{e^{\mathrm{i} w_{0}}-a_{m}}\left(\theta e^{\mathrm{i} w_{0}}+1-\theta\right) \\
& +\frac{K}{2} \widetilde{b}\left(\frac{\theta e^{\mathrm{i} w_{0}}\left(\theta e^{2 \mathrm{i} w_{0}}+1-\theta\right)}{a\left(e^{2 \mathrm{i} w_{0}}\right)}+2 \frac{\theta e^{\mathrm{i} w_{0}}+1-\theta}{a(1)}\right) .
\end{aligned}
$$


Thus applying the Neimark-Sacker bifurcation theorem [24], the stability of the closed invariant curve can be summarized as follows.

Theorem 2 If $c u_{*}>1$, then $u=u_{*}$ is asymptotically stable for any $\tau \in\left[0, \tau_{0}\right)$ and unstable for $\tau>\tau_{0}$. An attracting (repelling) invariant closed curve exists for $\tau>\tau_{0}$ if $\Re\left[e^{-\mathrm{i} w_{0}} C_{1}\left(\tau_{0}\right)\right]<$ $0(>0)$.

\section{Numerical simulations}

The purpose of this section is to test the results in Sections 2 and 3 by examples. Let $a=0.5$, $b=2, c=3.695$, then $u_{*}=0.5413 . c u_{*} \approx 2>1$ is satisfied. We compute the bifurcation points and direction of bifurcation at the fixed point of the numerical solutions of (1.1) with the NSFD- $\theta$ method (2.6) for some step-size. The results refer to Figures 1-4.

Firstly, let us see $\theta=0$. We obtain $h=1 / 2, \tau_{0}=1.8229 ; h=1 / 10, \tau_{0}=2.2950 ; h=1 / 40$, $\tau_{0}=2.3879$. The numerical solution refers to Figure 1 .

Secondly, let $\theta=1 / 4$. We obtain $h=1 / 2, \tau_{0}=2.2519 ; h=1 / 10, \tau_{0}=2.3653 ; h=1 / 40$, $\tau_{0}=2.4038$. The numerical solution is shown in Figure 2.

Thirdly, we test $\theta=1 / 2$. We obtain $h=1 / 2, \tau_{0}=2.7722 ; h=1 / 10, \tau_{0}=2.4302 ; h=1 / 40$, $\tau_{0}=2.4191$. The numerical solution refers to Figure 3 .
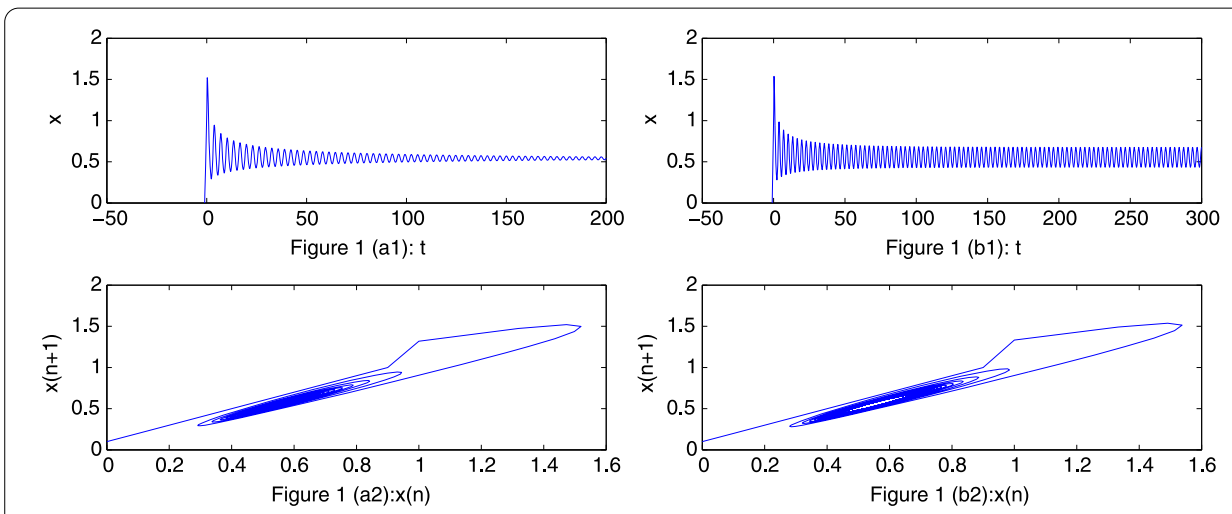

Figure 1 The numerical solution of (1.1) with the NSFD- $\theta$ method corresponding to $\theta=0, h=1 / 10$.

(a) $\tau=2.25$ and (b) $\tau=2.32$.
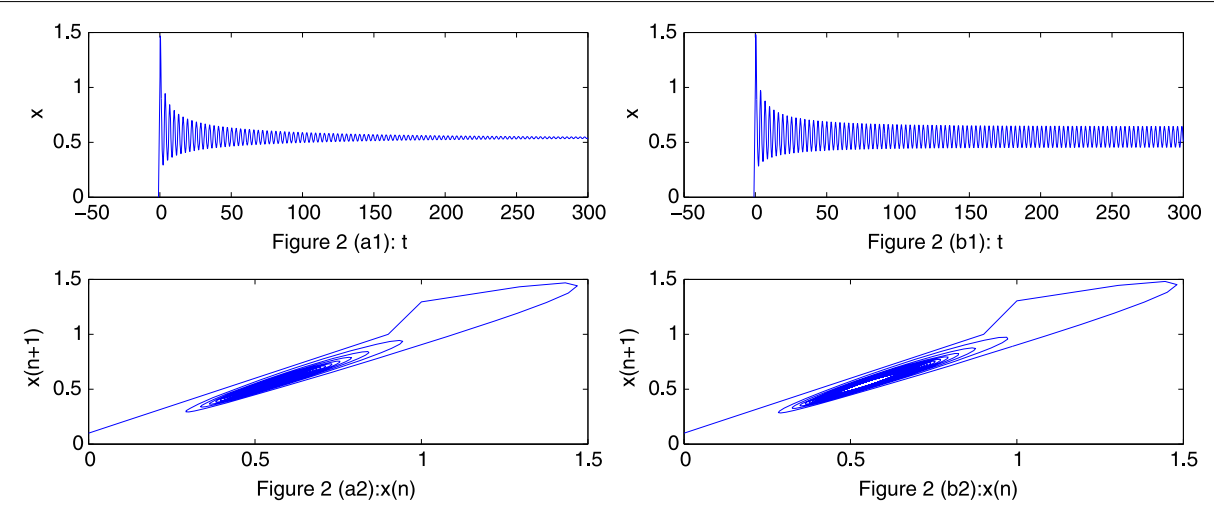

Figure 2 The numerical solution of (1.1) with the NSFD- $\theta$ method corresponding to $\theta=1 / 4, h=1 / 10$. (a) $\tau=2.32$ and (b) $\tau=2.4$. 

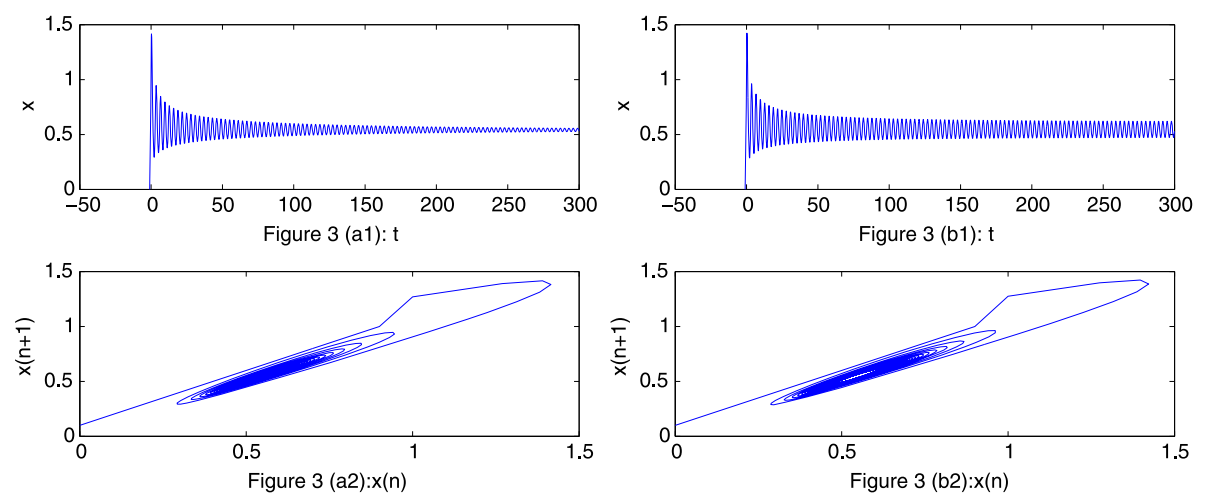

Figure 3 The numerical solution of (1.1) with the NSFD- $\theta$ method corresponding to $\theta=1 / 2, h=1 / 10$. (a) $\tau=2.4$ and (b) $\tau=2.45$.
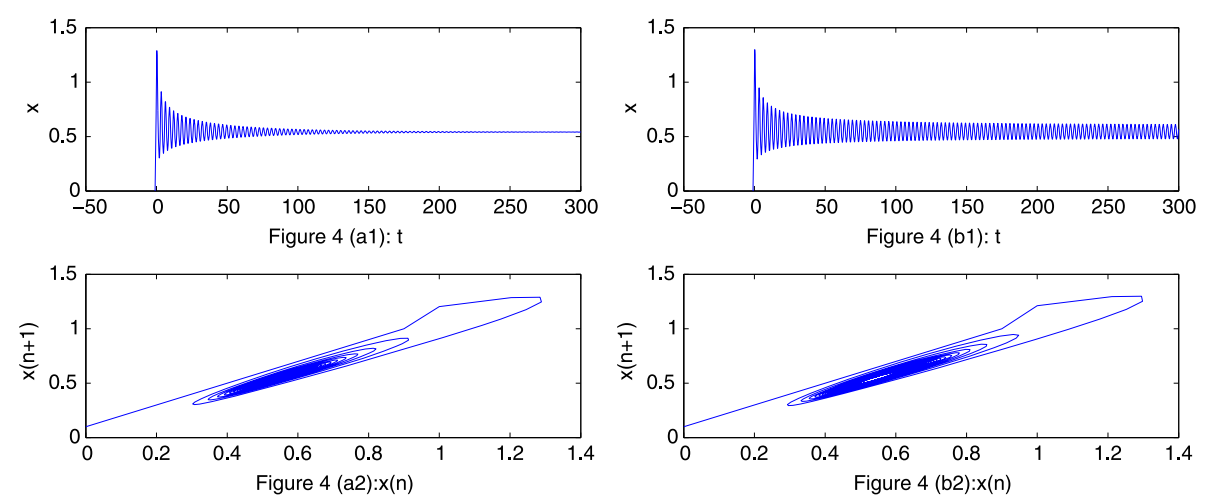

Figure 4 The numerical solution of (1.1) with the NSFD- $\theta$ method corresponding to $\theta=1, h=1 / 10$. (a) $\tau=2.45$ and (b) $\tau=2.55$.

Finally, let $\theta=1$. We have $h=1 / 2, \tau_{0}=2.7725 ; h=1 / 10, \tau_{0}=2.5346 ; h=1 / 40, \tau_{0}=$ 2.4483. The numerical solution is in Figure 4.

At the same time, by the Euler method we compute $h=1 / 2, \tau_{0}=1.4641 ; h=1 / 10, \tau_{0}=$ $2.1682 ; h=1 / 40, \tau_{0}=2.3526$. The numerical solution is in Figure 5 .

We see that there exists a sequence of $\tau_{i}$, and with the increasing of $m, \tau_{0}$ asymptotically converges to $\tau_{0}=2.4184$, which is the true value.

When $\theta=0,1 / 4,1 / 2,1$, we obtain Figures 1-4. From the above analysis and Figures 1-4, one comes to a better conclusion for $\theta=1 / 2$ than the other values by means of describing approximately the dynamics of the original system with the same step-size. Through Figure 5 we could argue that the NSFD- $\theta$ method is better than the Euler method by means of describing approximately the dynamics of the original system with the same step-size.

\section{Conclusions}

From the above analysis we could draw the biological conclusions. We can find that the delay does not influence the system's stability when the coefficients of the system satisfy the condition $c u_{*}<1$. But when the coefficients of system satisfy the condition $c u_{*}>1$, for a small delay the positive fixed point of system is stable, the number of red blood cells reaches an equilibrium. With the increasing of the delay (the critical point $\tau_{0}$ ), the positive 

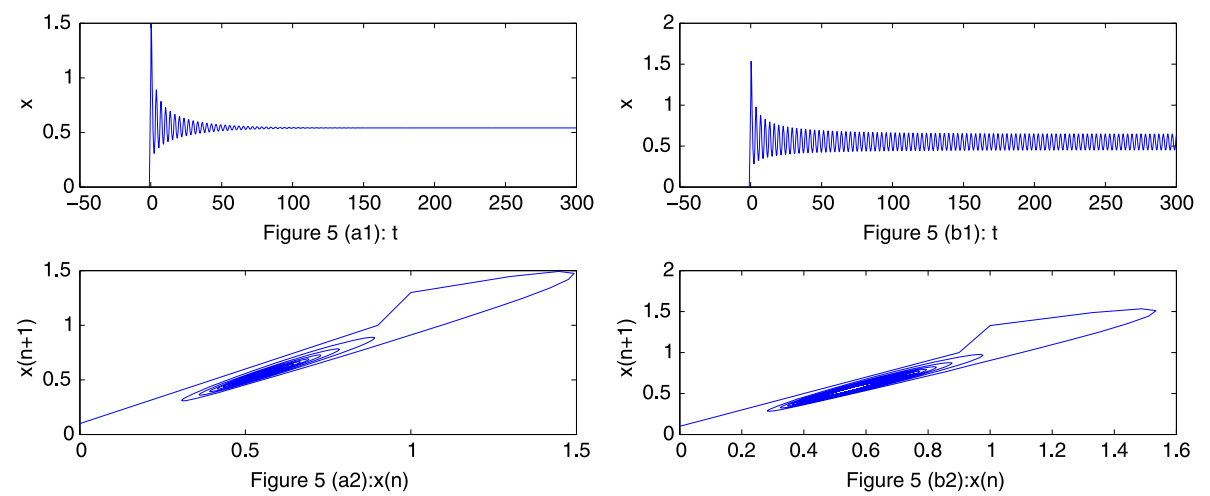

Figure 5 The numerical solution of (1.1) with the Euler method corresponding to $h=1 / 10$. (a) $\tau=2$ and (b) $\tau=2.2$.

fixed point loses its stability and a family of periodic solutions occurs, the number of red blood cells oscillates around the unstable equilibrium. In real life, we will try to control $\tau$ so that it does not exceed the critical point $\tau_{0}$. Therefore it can produce a stable system. These results are very useful to the biologists. The existence of a Neimark-Sacker bifurcation shows the periodic oscillatory behavior of the discrete red blood cell survival system.

It is noted that system (1.1) is a delay differential equation, making it an impossible task to obtain its analytical solutions to study its qualitative properties; therefore it is necessary to solve numerical solutions or approximate solutions of system (1.1) according to different discrete difference schemes. It is common to change a continuous dynamical system into a discrete-time dynamical system. However, the derived difference equation will be acceptable only if it preserves the dynamical feature of the continuous-time models. In this paper, through the NSFD- $\theta$ method, we obtain general results. Our results show the NSFD- $\theta$ method could inherit the Hopf bifurcation and the asymptotically stability for sufficiently small step-size. In particular, for $\theta=0,1 / 2,1$ the results hold for any step-size.

\section{Competing interests}

The author declares that they have no competing interests.

\section{Acknowledgements}

The author is grateful to the referees for their helpful comments and constructive suggestions. This work was supported by the National Natural Science Foundation of China (Grant No. 11401586) and by the Fundamental Research Funds for the Central Universities of China (14CX02159A) and by the NNSF Shandong Province (Nos. ZR2014AL008, ZR2014AQ004, ZR2014AQ014).

Received: 26 October 2014 Accepted: 2 March 2015 Published online: 14 March 2015

\section{References}

1. Wazewska-Czyzewska, M, Lasota, A: Mathematical problems of the dynamics of the red blood cells system. Rocz. Pol. Tow. Mat., 3 Mat. Stosow. 17, 23-40 (1976)

2. Wei, J, Li, MY: Hopf bifurcation analysis in a delayed Nicholson blowflies equation. Nonlinear Anal. 60, 1351-1367 (2005)

3. $\mathrm{Wu}, \mathrm{J}$ : Symmetric functional differential equations and neural networks with memory. Trans. Am. Math. Soc. 350, 4799-4838 (1998)

4. Song, Y, Wei, J, Han, M: Local and global Hopf bifurcation in a delayed hematopoiesis model. Int. J. Bifurc. Chaos 14, 3909-3919 (2004)

5. Song, YL, Wei, JJ, Yuan, Y: Bifurcation analysis on a survival red blood cells model. J. Math. Anal. Appl. 316(2), $459-471$ (2006)

6. Zhang, C, Zu, Y, Zheng, B: Stability and bifurcation of a discrete red blood cell survival model. Chaos Solitons Fractals 28, 386-394 (2006)

7. Ding, X, Fan, D, Liu, M: Stability and bifurcation of a numerical discretization Mackey-Glass system. Chaos Solitons Fractals 34, 383-393 (2007) 
8. Ding, X, Su, H: Dynamics of a discretization physiological control system. Discrete Dyn. Nat. Soc. 2007, 51406 (2007)

9. Wang, QB, Li, DS, Liu, MZ: Numerical Hopf bifurcation of Runge-Kutta methods for a class of delay differential equations. Chaos Solitons Fractals 42, 3087-3099 (2009)

10. Liu, MZ, Wang, QB: Numerical Hopf bifurcation of linear multistep methods for a class of delay differential equations. Appl. Math. Comput. 208, 462-474 (2009)

11. Wang, Y: Dynamics of a nonstandard finite-difference scheme for delay differential equations with unimodal feedback. Commun. Nonlinear Sci. Numer. Simul. 17, 3967-3978 (2012)

12. Zhang, L, Zhang, CJ, Zhao, DM: Hopf bifurcation analysis of integro-differential equation with unbounded delay. Appl. Math. Comput. 217, 4972-4979 (2011)

13. Wulf, $V$ : Numerical analysis of delay differential equations undergoing a Hopf bifurcation. Ph.D. thesis, University of Liverpool, Liverpool (1999)

14. Wulf, V, Ford, NJ: Numerical Hopf bifurcation for a class of delay differential equation. J. Comput. Appl. Math. 115, 601-616 (2000)

15. Mickens, RE: A nonstandard finite-difference scheme for the Lotka-Volterra system. Appl. Numer. Math. 45, 309-314 (2003)

16. Patidar, KC: On the use of nonstandard finite difference methods. J. Differ. Equ. Appl. 11(8), 735-758 (2005)

17. Roeger, LW, Lahodny, G Jr: Dynamically consistent discrete Lotka-Volterra competition systems. J. Differ. Equ. Appl. 19(2), 191-200 (2013)

18. Liao, C, Ding, X: Nonstandard finite difference variational integrators for nonlinear Schrödinger equation with variable coefficients. Adv. Differ. Equ. 2013, 12 (2013)

19. Tian, HJ, Kuang, JX: The stability of the $\theta$-methods in the numerical solution of delay differential equations with several delay terms. J. Comput. Appl. Math. 58, 171-181 (1995)

20. Ruan, $S$, Wei, J: On the zeros of transcendental functions with applications to stability of delay differential equations with two delays. Dyn. Contin. Discrete Impuls. Syst., Ser. A Math. Anal. 10, 863-874 (2003)

21. Hale, J: Theory of Functional Differential Equations. Springer, Berlin (1977)

22. Hassard, B, Kazarinoff, N, Wan, Y: Theory and Applications of Hopf Bifurcation. Cambridge University Press, Cambridge (1981)

23. Kuznetsov, YA: Elements of Applied Bifurcation Theory. Springer, New York (1995)

24. Wiggins, S: Introduction to Applied Nonlinear Dynamical System and Chaos. Springer, New York (1990)

\section{Submit your manuscript to a SpringerOpen ${ }^{\circ}$ journal and benefit from:}

- Convenient online submission

Rigorous peer review

- Immediate publication on acceptance

- Open access: articles freely available online

- High visibility within the field

- Retaining the copyright to your article 